\title{
Public health: the science of promoting health
}

\author{
Penka D. Gatseva • Mariana Argirova
}

Published online: 30 March 2011

(C) Springer-Verlag 2011

Public health is "the science and art of preventing disease, prolonging life and promoting health through the organized efforts and informed choices of society, organizations, public and private, communities and individuals" (Winslow 1920). The United Nations' World Health Organization defines health as "a state of complete physical, mental and social well-being and not merely the absence of disease or infirmity." (WHO 1946). Public health plays an important role in disease prevention efforts in both the developing world and in developed countries through local health systems and through international non-governmental organizations. Today, most governments recognize the importance of public health programs in reducing the incidence of disease, disability, and the effects of aging, although public health generally receives significantly less government funding compared to medicine.

This issue of the Journal of Public Health covers a broad range of current topics in public health; among them are the tobacco-related health problems. A number of scientific and medical evidence shows that the dangers associated with secondhand smoking are extremely serious and relevant. Many countries have implemented a full publicsmoking ban to protect the health of their citizens from the harmful effects of passive smoke. Balachandar et al. study the frequency of chromosomal aberrations among infertile

\section{P. D. Gatseva $(\square)$}

Department of Hygiene, Ecology and Epidemiology,

Medical University,

15a Vassil Aprilov Street,

4002 Plovdiv, Bulgaria

e-mail: gatseva_p@mail.bg

\section{Argirova}

Department of Chemistry and Biochemistry, Medical University, 15a Vassil Aprilov Street,

4002 Plovdiv, Bulgaria male subjects in comparison to control subjects with the same pack-years and their offsprings (secondhand smokers). The authors identify higher degrees of chromosomal damage in infertile subjects compared to control smokers but $\mathrm{Y}$ chromosomal alterations were found in both subjects and offsprings. This finding is a distress signal for possible transmission of the current health-related problems of smokers into the next generation.

Health hazards of obesity and being overweight are a growing concern for public health professionals. A study by $\mathrm{Bau}$ al. points out that postmenarche girls are more than twice as likely to be overweight and/or abdominally overweight as premenarche girls within the same age group. Therefore, not only the age but also the progress of maturation is important for assessing the growth and health of adolescents.

For a long time obesity was considered a problem in high-income countries but now it is also growing in lowincome regions, especially in urban settings (James et al. 2001). A cohort study by Shiying Fu et al. examines the influence of central obesity indexed by waist circumference to height ratio $(\mathrm{W} / \mathrm{H})$ on the adverse levels of metabolic syndrome among adults with normal weight in a lowincome rural Chinese region. Groups adjusted for age and sex with $\mathrm{W} / \mathrm{H}$ index below 0.5 have significantly different levels of blood pressure, triglycerides, high-density lipoprotein-bound cholesterol, and glucose compared to the group with $\mathrm{W} / \mathrm{H}$ index above 0.5 . The findings from the study underscore the importance of the utility of $\mathrm{W} / \mathrm{H}$ as a measure of abdominal fat accumulation in predicting metabolic syndrome even among individuals with normal weight in terms of body mass index.

During the last years, a dramatic increase in the average lifespan is widely credited to public health achievements such as vaccination programs and control 
of infectious diseases, effective safety policies such as motor-vehicle and occupational safety, improved family planning, chlorination of drinking water, smoke-free measures, and programs designed to decrease chronic diseases. Kirnpal-Kaur Banga Singh et al. report on a 9-year study on shigellosis, the highest prevalence of which is observed in pediatric patients of a hospital in northeastern region of Malaysia. The susceptibility of all isolated Shigella strains was tested against seven antibiotics. Ceftriaxone, ciprofloxacin, and nalidixic acid were the most effective against the Shigella strains, whereas tetracycline and trimethoprim-sulfamethoxazole exhibited high frequencies of resistance. The results obtained are helpful for pediatricians and microbiologists in the selection of appropriate antibiotics for the management of diarrhea, and thus, are also helpful in the reduction of the morbidity and mortality associated with Shigella spp. infection.

Iodine deficiency is another global public health problem and iodine supplementation is probably the cheapest and most effective method of prevention. The Bulgarian National Strategy for Prevention and Control of Iodine Deficiency Disorders (IDD) was developed in 1994, and regular surveys undertaken in 2000-2003 indicated a normalization of the iodine supply of Bulgarian population. A study by Gatseva et al. evaluates the iodine status of some at-risk population groups (schoolchildren and pregnant women) living in an iodine-deficient area in Bulgaria. Data show that almost one third of the inspected children have iodine deficiency, and median urinary iodine concentration found for pregnant women is an indicator of insufficient iodine intake. This information sounds an alarm for more targeted prevention strategies for the population groups at risk.

Osteoporosis is a disease affecting millions of people all over the world. By learning the risk factors for development of osteoporosis it can be turned into a preventable and treatable disease. Omasu et al. present a study revealing that calcaneus stiffness index in adult premenopausal females is strongly influenced by the amount of physical activity during puberty, especially during senior high school. Based on the negative correlation between stiffness index and urinary deoxypyridinoline (DPD) as an index of bone metabolism, the authors hypothesize that there may be a mechanism by which physical activity during one's high school years lowers DPD concentrations, thereby suppressing bone resorption and increasing bone mass. In other words, the prevention of osteoporosis should start in adolescence.

In this issue, the reader will find a study by Kobayashi et al. on Japanese community pharmacists' attitudes toward generic-substitution policy implementation. The issue is topical considering the annual increase in the total prescription drug costs in many countries, including Japan. The authors identify the major obstacles for switching from brand to generic pharmaceuticals and conclude that it is not common for Japanese community pharmacists to recommend generic substitution to patients in spite of their positive attitudes towards generic substitution.

Though it has long been known that poor health and unemployment often go together, the Hollederer's microcensus analyses reveal the need for a specification of target groups, strategies for making contact, and all-inclusive concepts to promote good health. The results on the health of the children of unemployed principal income earners also argue in favor of approaches that systemically address the entire family. Chances for reintegration are substantially lower for persons with a disability. The author concludes that microcensus analysis is very suitable for identifying groups of persons at elevated risk of morbidity and has the potential for development as a source of a continuous, systematic tool for meeting the challenges of public health.

Rösler-Schidlack et al. focus their research on a littlestudied group of primary family caregivers. The conclusion of their survey is that caregivers in midlife, especially caregiving women, are at-risk groups for poor health. The authors recommend different kinds of measures that could reduce the health burden of caregiving women: instrumental support and information on caregiving tasks, combined with health-promoting interventions and regular respite.

In conclusion, the readers of this issue will expand their knowledge on the present challenges for public health professionals and identify the multifaceted challenges of the future.

\section{References}

James PT, Leach R, Kalamara E, Shayeghi M (2001) The worldwide obesity epidemic. Obes Res 9:S228-S233

WHO (1946) Definition of health. Preamble to the Constitution of the World Health Organization as adopted by the International Health Conference. WHO, Geneva

Winslow C-EA (1920) The untilled fields of public health. Science 51:23 